\title{
IEEJ Industry Applications Society Program Committee
}

\section{IEEJ Transactions on Industry Applications (in Japanese)}

Editor in Chief: Toshiyuki Murakami, Keio University

Vice Chief: Satoshi Suzuki, Tokyo Denki University

Semiconductor Power Converter Subcommittee (D1)

Chairperson: Kazuaki Mino, Murata Manufacturing, Vice Chairperson: Toshimitsu Morizane, Osaka Institute of Technology

Secretaries: Jun-ichi Itoh, Nagaoka University of Technology, Nobukazu Hoshi, Tokyo University of Science, Sari Maekawa, Toshiba, and Keiji Wada, Tokyo Metropolitan University

Industrial Instrumentation and Control, Mechatronics Control Subcommittee (D2)

Chairperson: Satoshi Komada, Mie University, Vice Chairperson: Yasue Mitsukura, Keio University

Secretaries: Takenori Atsumi, HGST Japan, Yutaka Uchimura, Shibaura Institute of Technology, and Hiroshi Fujimoto, The University of Tokyo

Rotating Machinery, Motor Drive, Linear Drives, Subcommittee (D3)

Chairperson: Isao Hirotsuka, Chubu University, Vice Chairperson: Kichiro Yamamoto, Kagoshima University

Secretaries: Kan Akatsu, Shibaura Institute of Technology, Yuji Enomoto, Hitachi, Yasuaki Sakamoto, Railway Technical Research Institute, Mimpei Morishita, Kogakuin University, Shu Yamamoto, Polytechnic University, and Yasuhiro Yamamoto, Meidensha

Vehicle Technology, Intelligent Transport Systems, Home and Consumer Appliances Subcommittee (D4)

Chairperson: Kazuaki Yuki, Toshiba, Vice Chairperson: Masafumi Miyatake, Sophia University

Secretaries: Masayuki Sanada, Osaka Prefecture University and Satoshi Takahashi, Nagoya Electric Works

Monozukuri, Innovative Industrial System, Transportation and Electric Railway, Smart Facilities, Public Plant

Engineering Subcommittee (D5)

Chairperson: Kazuaki Yuki, Toshiba, Vice Chairperson: Masafumi Miyatake, Sophia University

Secretaries: Norimitsu Ichikawa, Kogakuin University, Teruo Usami, Kyoto Gakuen University, Takafumi Koseki, The University of Tokyo, Kenji Suzuki, University of Tsukuba, and Osamu Yamanaka, Toshiba

\section{IEEJ Journal of Industry Applications}

Editor in Chief: Kiyoshi Ohishi, Nagaoka University of Technology

Power Electronics and Its Applications (D6)

Chairperson: Tomoki Yokoyama, Tokyo Denki University, Vice Chairperson: Hideaki Fujita, Tokyo Institute of Technology

Secretary: Hitoshi Haga, Nagaoka University of Technology

Motion Control, Robotics, Sensing and their Applications (D7)

Chairperson: Masaaki Shibata, Seikei University, Vice Chairperson: Yasutaka Fujimoto, Yokohama National University

Secretary: Jun Ishikawa, Tokyo Denki University, Tadanao Zanma, Chiba University, and Hiroshi Fujimoto, The University of Tokyo

Electric Machine, Motor Drive and their Applications (D8)

Chairperson: Hisao Kubota, Meiji University, Vice Chairperson: Takashi Kosaka, Nagoya Institute of Technology

Secretary: Keiichiro Kondo, Chiba University, Masayuki Sanada, Osaka Prefecture University, and Akio Toba, Fuji Electric 